\title{
Efficacy of rescue blanket versus conventional blanket in terms of preventing accidental hypothermia during patient transfer by emergency medical service: a randomized clinical trial
}

\author{
Peyman Saberian ${ }^{1,2}$, Mostafa Sadeghi ${ }^{3}$, Parisa Hasani-Sharamin ${ }^{4}$, Maryam Modaber $^{4}$, Amirreza Farhoud \\ Mehrad Aghili, ${ }^{1,6^{*}}$
}

'Prehospital and Hospital Emergency Research Center, Tehran University of Medical Sciences, Tehran, Iran ${ }^{2}$ Department of Anesthesiology, Imam Khomeini Hospital Complex, Tehran University of Medical Sciences, Tehran, Iran ${ }^{3}$ Department of Anesthesiology, Shariati Hospital, Tehran University of Medical Sciences, Tehran, Iran ${ }^{4}$ Tehran Emergency Medical Service Center, Tehran, Iran

${ }^{5}$ Reconstruction Research Center, Department of Orthopedic Surgery, Imam Khomeini Hospital Complex, Tehran University of Medical Sciences, Tehran, Iran

${ }^{6}$ Department of Emergency Medicine, Shariati Hospital, Tehran University of Medical Sciences, Tehran, Iran

\author{
Received: 6 October 2019 \\ Accepted: 17 December 2019 \\ Published online: 27 December \\ 2019 \\ *Corresponding author: Mehrad \\ Aghili; Department of Emergency \\ Medicine, Shariati Hospital, Tehran \\ University of Medical Sciences, \\ Tehran, Iran. Email: aghili_m@sina. \\ tums.ac.ir \\ Competing interests: None. \\ Funding information: This study \\ was supported by a grant (18192 \\ 164316 ) received from Tehran \\ Emergency Medical Service Center. \\ Citation: Saberian P, Sadeghi M \\ Hasani-Sharamin P, Modaber M \\ Farhoud A, Aghili M. Efficacy of \\ rescue blanket versus conventional \\ blanket in terms of preventing \\ accidental hypothermia during \\ patient transfer by emergency \\ medical service: a randomized \\ clinical trial. Journal of Emergency \\ Practice and Trauma 2020; 6(1): 38- \\ 42. doi: 10.15171/jept.2019.27.
}

\begin{abstract}
Objective: This study aimed to compare the efficacy of rescue blankets with conventional blankets in terms of preventing hypothermia in the pre-hospital setting.

Methods: In this randomized clinical trial, patients older than 18 years old with Cold Discomfort Scale (CDS) $>2$, and those who were transferred to the emergency department (ED) by emergency medical service entered the study. Patients were randomly divided into two groups based on the type of transfer. In this regard, one group was transferred with rescue blankets and the other group was transferred with conventional blankets. The tympanic temperature in patients and CDS were recorded before the use of blanket (primary) and at the time of arrival in the ED (secondary).

Results: Finally, 161 patients with the mean age of $45.31 \pm 19.8$ years were included $(63.4 \%$ were male). Totally, 88 cases (54.7\%) were transferred with rescue blankets and 73 cases (45.3\%) with conventional blankets. The mean of the primary tympanic temperatures in the rescue and conventional blanket groups were $36.20 \pm 0.84^{\circ} \mathrm{C}$ and $36.34 \pm 0.65^{\circ} \mathrm{C}$, respectively $(P=0.23)$. The mean of the primary CDS in rescue and conventional blanket groups were $6.55 \pm 1.95$ and $5.89 \pm 2.29$, respectively $(P=0.05)$. Also, the mean of the secondary tympanic temperatures in the rescue and conventional blanket groups were $36.59 \pm 0.47^{\circ} \mathrm{C}$ and $36.76 \pm 0.48^{\circ} \mathrm{C}$, respectively $(P=0.03)$. Besides, the mean of the secondary CDS in the rescue and conventional blanket groups were $2.64 \pm 2.80$ and $2.41 \pm 1.29$, respectively $(P=0.48)$. Conclusion: According to the results, there is no significant difference in the tympanic temperature and CDS of the patients transferred with the rescue blanket compared with the conventional blanket.

Keywords: Blanket, Emergency medical service, Hypothermia, Prevention, Randomized clinical trial
\end{abstract}

\section{Introduction}

Random hypothermia is defined as lowering the body temperature below $35^{\circ} \mathrm{C}$ without initial defects in the temperature system of the body. Accidental hypothermia may occur in pre-hospital conditions and may complicate pre-hospital emergency care (1). Considering the severity and mechanism of injury, the occurrence of shock, the administration of intravenous fluids, and the duration of rescuing the injured, the incidence of hypothermia in patients can range from $1.6 \%$ to $43 \%$, (1-3). Loss of heat may occur at the scene of the incident as well as during the transfer. Hypothermia can occur not only for patients outdoors but also for patients at home (4). For pre-hospital personnel, it is very important to prevent the loss of body heat in pre-hospital care. However, exposure to cold is often an ignored problem in pre-hospital care (5). When hypothermia occurs, considerable efforts are needed to warm up. The treatment of hypothermia 
generally involves passive rewarming, the most basic of which is the patient's transfer from the cold environment and the use of blankets or other insulating materials to maintain body heat $(6,7)$. In many cases, where the patient can be transferred quickly to a care facility, the passive rewarming approach is all that has been mentioned in the pre-hospital setting. Many studies have been performed to evaluate and compare different materials and products (711). However, recommendations concerning which items should be used in the field are often based on experience instead of scientific evidence. There are also limited studies on the treatment methods and the availability of suitable equipment for protecting against cold. One study showed that insulating and protecting from cold in pre-hospital care is very important, and also pointed out that polyester blankets that are used in ambulances are ineffective in windy conditions (7). Since January 2018, the Iranian rescue blanket has been used for patients in ambulances of Tehran Emergency Medical Service center. However, regarding the importance of hypothermia management in the pre-hospital setting, a study was conducted to evaluate the effectiveness of rescue blankets and compared them with conventional blankets. Considering today's requirements of evidence-based medicine and helping pre-hospital guidelines on protecting against cold in prehospital settings, the present study aims to investigate the current equipment used to prevent hypothermia in the pre-hospital setting and compare rescue blankets (thermal insulation) with conventional blankets made of polyester in different atmospheric conditions.

\section{Methods}

A randomized clinical trial was designed and registered with IRCT20190416043288N1 code at the Iranian Clinical Trial Registration Center. This study was undertaken in pre-hospital emergency stations in 4 regions of Tehran in 2018. Rescue blankets and thermometers were distributed in each region. Required training was given to the prehospital emergency personnel. Questionnaires were collected under ethical principles and the confidentiality of information was taken into account. The checklists were anonymous and the information remained secret. The researchers adhered to the principles of Helsinki Declaration, and patients entered the study after their consent was obtained.

In this study, the inclusion criteria were patients older than 18 years who were transferred to the hospital via the pre-hospital emergency service, and had Cold Discomfort Scale $(\mathrm{CDS})>2$. The exclusion criteria were GCS $<15$, arriving at the hospital in less than 10 minutes, being transferred via air medical service, being susceptible to primary hypothermia due to underlying diseases causing disruptions in core body temperature (such as metabolic disorders, medications, sepsis), having a history of skin problems or CNS disorder. According to the study of Aléx et al (4), assuming a mean of 4.2 for CDS, 1.78 SD, 95\%
CI, and $80 \%$ study power, the minimum sample size in each group was 100 individuals. Pre-hospital emergency stations were randomly divided for intervention based on the use of conventional (polyester) or rescue blankets. Three stations used conventional blankets and three stations used rescue blankets in the transference of patients to the hospital. Therefore, patients were randomly divided into two groups, one group with conventional blankets made of polyester and the other one with rescue blankets. Demographic information, weather conditions during the transference, the type of blanket used, duration of blanket use, and patient's vital signs were collected based on the data registry form. The tympanic temperature and the patient's CDS were recorded 2 times: before the blanket was used (primary tympanic temperature and CDS) and at the time of arrival in the ED of the destination hospital (secondary tympanic temperature and CDS). Pre-hospital emergency personnel completed all checklists.

The required data were extracted in the Excel format from the pre-hospital registry bank and after filling out the incomplete data from the records, SPSS software version 22 was used for statistical analysis. Independent $t$ test was used to compare tympanic temperatures, CDSs, blood pressures, and pulse rates. Univariate test was used to control the effect of weather condition types on tympanic temperature changes. The significance level was considered $5 \%$ in all tests.

\section{Results}

In this study, 211 patients entered in which 39 cases were excluded according to the exclusion criteria and 11 cases declined to participate. Finally, 161 patients with a mean age of $45.31 \pm 19.8$ years were included. The CONSORT flowchart of the study is shown in Figure 1.

Out of 161 patients studied, 102 (63.4\%) were male and 59 (36.6\%) were female; 88 (54.7\%) patients and 73 (45.3\%) patients were transferred with rescue blankets and conventional blankets, respectively. The mean ambient temperature was $16.37 \pm 3.43{ }^{\circ} \mathrm{C}$. The mean transfer time to the hospital was $18.23 \pm 9.43$ minutes. It was sunny in 102 cases (63.4\%) and non-sunny in 59 cases (36.6\%). Out of 161 patients, only 8 (5\%) had wet clothing. Regarding the chief complaints, there were 70 (43.48) traumas, 28 (17.39) cardiac problems, 14 (8.7) respiratory problems, 9 (5.59) strokes, and 40 (24.84) other problems. Basic information of participants is shown in Table 1. The mean of the primary tympanic temperature for the rescue blanket group was $36.20 \pm 0.84^{\circ} \mathrm{C}$ and it was $36.34 \pm 0.65^{\circ} \mathrm{C}$ $(P=0.23)$ for the conventional blanket group. The mean of the primary CDS score in the first group was $6.55 \pm 1.95$ and in the second one, it was $5.89 \pm 2.29(P=0.051)$.

The variables evaluated at the time of arrival in the emergency department (ED) of the destination hospital for two groups are reported in Table 2. The mean of the secondary tympanic temperatures (at the time of patient's arrival in the ED of the hospital) in the rescue blanket 


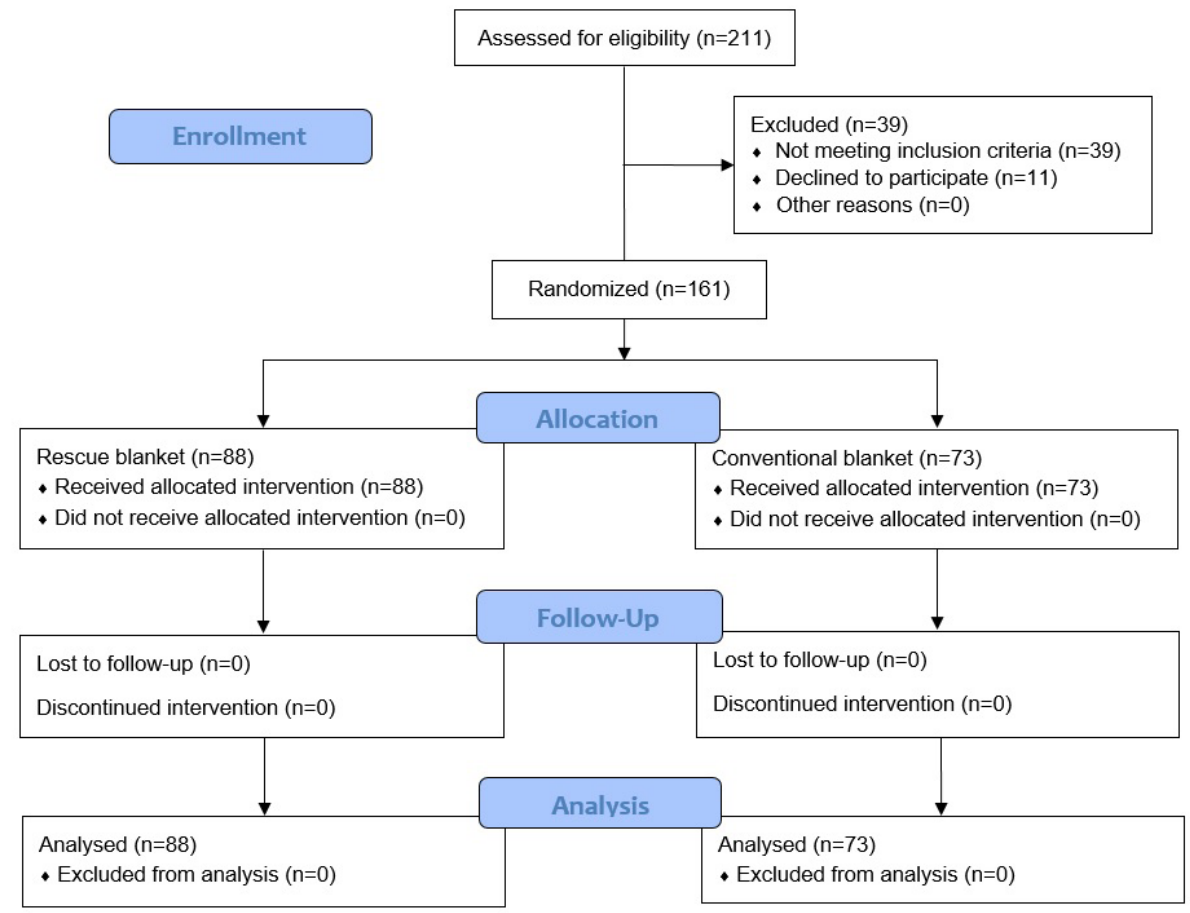

Figure 1. CONSORT flowchart of enrolled patients.

and the conventional blanket groups were $36.59 \pm 0.47^{\circ} \mathrm{C}$ and $36.76 \pm 0.48{ }^{\circ} \mathrm{C}$, respectively $(P=0.03)$. After using blankets, the CDS was $2.64 \pm 2.80$ and $2.41 \pm 1.29$ in the rescue and conventional blanket groups, respectively $(P=$ 0.48). There was no significant difference in pulse rates, blood pressures and SPO2s between the two groups after using blankets.

Table 1. Basic information of participants

\begin{tabular}{lccc}
\hline \multirow{2}{*}{ Variable } & $\begin{array}{c}\text { Rescue } \\
\text { Blanket }(\mathbf{n}=\mathbf{8 8})\end{array}$ & $\begin{array}{c}\text { Conventional } \\
\text { Blanket }(\mathbf{n}=\mathbf{7 3})\end{array}$ & $\begin{array}{c}\boldsymbol{P} \\
\text { value }\end{array}$ \\
\cline { 2 - 4 } & \multicolumn{2}{c}{ Mean \pm SD } & 0.23 \\
\hline Tympanic temperature & $36.20 \pm 0.84$ & $36.34 \pm 0.65$ & 0.051 \\
\hline Cold discomfort scale & $6.55 \pm 1.95$ & $5.89 \pm 2.29$ & 0.051 \\
\hline Pulse rate & $81.46 \pm 11.72$ & $83.33 \pm 10.44$ & 0.32 \\
\hline Blood pressure & $116.29 \pm 24.03$ & $121.56 \pm 27.21$ & 0.23 \\
\hline O2 saturation & $97.00 \pm 1.58$ & $96.18 \pm 4.3$ & 0.12 \\
\hline
\end{tabular}

Table 2. Variables evaluated at the time of patient arrival in the ED of the destination hospital

\begin{tabular}{lccc}
\hline \multirow{2}{*}{ Variable } & $\begin{array}{c}\text { Rescue } \\
\text { Blanket }(\mathbf{n}=\mathbf{8 8})\end{array}$ & $\begin{array}{c}\text { Conventional } \\
\text { Blanket }(\mathbf{n}=\mathbf{7 3})\end{array}$ & $\begin{array}{c}\boldsymbol{P} \\
\text { value }\end{array}$ \\
\cline { 2 - 3 } & \multicolumn{2}{c}{ Mean \pm SD } & \\
\hline Tympanic temperature & $36.59 \pm 0.47$ & $36.76 \pm 0.48$ & 0.03 \\
Cold discomfort scale & $2.64 \pm 2.80$ & $2.41 \pm 1.29$ & 0.48 \\
Pulse rate & $81.25 \pm 10.57$ & $82.60 \pm 8.80$ & 0.39 \\
\hline Blood pressure & $119.54 \pm 19.76$ & $121.28 \pm 20.65$ & 0.58 \\
\hline O2 saturation & $96.56 \pm 3.26$ & $96.43 \pm 3.80$ & 0.81 \\
\hline
\end{tabular}

At the beginning of the study, there was no significant difference concerning the primary tympanic temperatures between the two groups. CDS scores did not have a significant difference after the use of blankets in the two groups. Using the blankets, secondary tympanic temperatures in both groups were significant. Based on the univariate test, the mean difference of the secondary tympanic temperatures for the conventional and rescue blankets, and the effect of weather (sunny or nonsunny) were calculated indicating a significant difference in the interaction between the two layers (group and weather) $(P=0.045)$. Although the independent effect of weather $(P=0.98)$ and blanket type $(P=0.11)$ on the secondary tympanic temperature were not significant, the interaction effect of the two on the secondary tympanic temperature was meaningful. After controlling the impact of ambient temperature on the tympanic temperature in the two groups (through using the univariate test), it was found that there was no significant difference in the type of blankets on the secondary tympanic temperatures. In terms of satisfaction, 59 patients $(36.6 \%)$ evaluated rescue blankets as appropriate, 72 patients $(44.7 \%)$ found conventional blankets to be more appropriate, and 30 patients (18.7\%) expressed no difference between the two types of blankets.

\section{Discussion}

According to the results of this study, no significant difference was observed in vital signs and cold sensation based on CDS of patients in the rescue blanket group in 
comparison to the conventional blanket group. However, using conventional blankets in sunny weather, and rescue blankets in windy and rainy weather can increase the body's core temperature. In a study by Jussila et al, it was shown that choosing the optimal rescue bag made of water-resistant layers could provide adequate protection against cold, wind, and splash of water during the pre-hospital transfer. This finding is consistent with the results of our study (10). A systematic review was conducted by Haverkamp et al (12) regarding current hypothermia treatment and their efficacy in pre-hospital hypothermia management. In this systematic review, 913 articles were retrieved in which 51 articles had a focus on either passive thermal or active heating. The most effective thermal insulation system was the combination of thermal insulation and vapor barrier. The results of this study indicated that in patients with mild hypothermia and without active rewarming, the use of both blankets increased the temperature and improved the patient's cold sensation. In a study by Thomassen et al (13), three methods for preventing randomized hypothermia were compared. In this study, Hibler's method, bubble wrap and ambulance blankets were compared. After 15 minutes, by Hibler's method in comparison to wrapping with ambulance blankets or bubble wrap, skin temperature was significantly higher. There were no differences in core temperature between the three insulating methods. Hibler's method was the participants' preferred method for preventing hypothermia. This study showed that the combination of a vapor-tight layer and an additional dry insulating layer (Hibler's method) is the most effective wrapping method to prevent heat loss, as demonstrated by increased skin temperatures, lower metabolic rate, and better thermal comfort. In our study, rescue blankets are more helpful in preserving body heat and preventing hypothermia in rainy weather. It seems that the simultaneous use of the conventional blankets and then rescue blankets can prevent hypothermia, especially on rainy days. Further research in this regard is recommended.

\section{Limitations}

In this study, the effect of the season has not been considered. Therefore, it is better to conduct studies in the winter or in cold and rainy weather in order to compare results. The climate classifications in this research were sunny and non-sunny days. It is better to consider air temperature and rainy or sunny weather independently.

\section{Conclusion}

According to the results of this study, no significant difference was observed in vital signs and cold sensation based on CDS between the rescue blanket and conventional blanket groups. However, using a conventional blanket in sunny weather and rescue blanket in windy and rainy weather increased the body's core temperature. On the other hand, since there is reduced risk of infection transmission in rescue blankets, as they are disposable, and the fact that they occupy less space in the ambulance jump bag, besides it is easy to carry them in difficult conditions; it seems that the use of rescue blankets is more ideal in the pre-hospital setting.

\section{Authors' contributions}

Conception or design of the work: PS, MS, and PHS; Data acquisition: PHS and MM; Analysis and interpretation of data for the work: AF and MA; Drafting the work: PHS, MM and MA; Revising it critically for important intellectual content: PS, MS, AF; Final approval of the version to be published: All the authors; Agreement to be accountable for all aspects of the work in ensuring that questions related to the accuracy or integrity of any part of the work are appropriately investigated and resolved: All the authors.

\section{Ethical issues}

The study protocol was approved by the ethics committee of Tehran University of Medical Sciences (IR.TUMS.VCR. REC.1397.1092).

\section{Acknowledgements}

We would like to express our thanks to hospitals and emergency medical service staff who helped us in conducting this study. In addition, we sincerely express our gratitude to the Pre-hospital and Hospital Emergency Research Center for the supports provided.

\section{References:}

1. Ireland S, Endacott R, Cameron P, Fitzgerald M, Paul E. The incidence and significance of accidental hypothermia in major trauma--a prospective observational study. Resuscitation 2011; 82(3): 300-6. doi: 10.1016/j. resuscitation.2010.10.016.

2. Beilman GJ, Blondet JJ, Nelson TR, Nathens AB, Moore FA, Rhee P, et al. Early hypothermia in severely injured trauma patients is a significant risk factor for multiple organ dysfunction syndrome but not mortality. Ann Surg 2009; 249(5): 845-50. doi: 10.1097/ SLA.0b013e3181a41f6f.

3. Martin RS, Kilgo PD, Miller PR, Hoth JJ, Meredith JW, Chang MC. Injury-associated hypothermia: an analysis of the 2004 National Trauma Data Bank. Shock 2005; 24(2): 114-8. doi: 10.1097/01.shk.0000169726.25189.b1.

4. Aléx J, Karlsson S, Saveman BI. Patients' experiences of cold exposure during ambulance care. Scand J Trauma Resusc Emerg Med 2013; 21: 44. doi: 10.1186/1757-724121-44.

5. Lintu NS, Mattila MA, Holopainen JA, Koivunen M, Hanninen OO. Reactions to cold exposure emphasize the need for weather protection in prehospital care: an experimental study. Prehosp Disaster Med 2006; 21(5): 316-20. doi: 10.1017/s1049023x00003940. 
6. Giesbrecht GG. Prehospital treatment of hypothermia. Wilderness Environ Med 2001; 12(1): 24-31. doi: 10.1580/1080-6032(2001)012[0024:ptoh]2.0.co;2.

7. Henriksson O, Lundgren JP, Kuklane K, Holmer I, Bjornstig U. Protection against cold in prehospital carethermal insulation properties of blankets and rescue bags in different wind conditions. Prehosp Disaster Med 2009; 24(5): 408-15. doi: 10.1017/s1049023x00007238.

8. Light IM, Norman JN. The thermal properties of a survival bag incorporating metallised plastic sheeting. Aviat Space Environ Med 1980; 51(4): 367-70.

9. Henriksson O, Bjornstig U, Saveman BI, Lundgren PJ. Protection against cold - a survey of available equipment in Swedish pre-hospital services. Acta Anaesthesiol Scand 2017; 61(10): 1354-60. doi: 10.1111/aas.13002.

10. Jussila K, Rissanen S, Parkkola K, Anttonen H. Evaluating cold, wind, and moisture protection of different coverings for prehospital maritime transportation-a thermal manikin and human study. Prehosp Disaster Med 2014; 29(6): 580-8. doi: 10.1017/s1049023x14001125.

11. Cohen S, Hayes JS, Tordella T, Puente I. Thermal efficiency of prewarmed cotton, reflective, and forced-warm-air inflatable blankets in trauma patients. Int J Trauma Nurs 2002; 8(1): 4-8. doi: 10.1067/mtn.2002.121312.

12. Haverkamp FJC, Giesbrecht GG, Tan E. The prehospital management of hypothermia - An up-to-date overview. Injury 2018;49(2):149-64. doi: 10.1016/j. injury.2017.11.001.

13. Thomassen $\varnothing$, Færevik $H$, Østerås $\varnothing$, Sunde GA, Zakariassen E, Sandsund M, et al. Comparison of three different prehospital wrapping methods for preventing hypothermia--a crossover study in humans. Scand J Trauma Resusc Emerg Med 2011; 19: 41. doi: 10.1186/1757-7241-19-41. 